\title{
Tracheal remodelling supports stem cells
}

\author{
The vascular system, plastic and positioned in the vicinity of tissues, is an undervalued regulator of adult stem \\ cells. Two studies now show that the vascular-like Drosophila trachea is reshaped after intestinal damage or \\ tumour formation and that this remodelling is required for compensatory intestinal stem cell proliferation and \\ tumour growth.
}

\section{Louis Gervais and Allison J. Bardin}

( uring homeostasis, adult stem cells of many tissues are quiescent or slowly dividing, compensating for differentiated cell turnover. After damage, these stem cells often offset cell loss by increasing their proliferation rate. Activation of stem cell proliferation involves not only cell intrinsic factors but also extrinsic signals from surrounding cells of the tissue. Inter-tissue communication contributes actively to allow the adaptation of adult stem cells to different tissue contexts such as stress or damage. Because of its role in gas and fluid delivery, the vascular system is in close proximity to many adult stem cells and seems to be involved in crafting their microenvironment ${ }^{1-3}$. Similarly, neovascularization participates in pathological states such as inflammation and tumorigenesis ${ }^{4}$. Despite the recognised importance of the vascular system, specific roles of induced vascular remodelling in tissue regeneration, their mechanisms and how they relate to neo-angiogenesis in tumorigenic contexts remain unclear. Indeed, in vivo experimental systems used to study the interaction between adult stem cells and the vascular system in the context of tissue homeostasis are still rare, and ex vivo organoid cultures do not fully recapitulate the complexity of tissue environments. Two related studies published in Nature Cell Biology from Perochon et al. and Tamamouna et al. use Drosophila adult intestinal regeneration as a model to investigate the intestinal co-regulation with the tracheal system, an equivalent of the mammalian vascular system (Fig. 1) (5,6 $^{5}$.

The adult intestinal epithelium of Drosophila is pseudostratified and comprised of differentiated cells, namely enterocytes (EC) and enteroendocrine cells, which are renewed by intestinal stem cells (ISCs) ${ }^{7}$. Damage, infection or oxidative stress induces ISC proliferation to compensate for cell death and return to tissue homeostasis. ISC regulation relies on the concerted action of many signals from dying cells of the tissue, visceral muscles surrounding the epithelium, neurons innervating the tissue and systemic factors ${ }^{7}$. Little is known about the role of the intestinal trachea in the regulation of ISC behaviour, though a possible function in ISC maintenance has been proposed previously ${ }^{8}$. Earlier work also demonstrated that terminal tracheal cells (TTCs) associated with the gut change their organisation under neuronal control in response to nutritional cues ${ }^{9}$. TTCs equivalent to mammalian leading vascular cells called tip cells, found at the periphery of the general tracheal system in target tissues ${ }^{10}$. TTCs have a branched morphology, projecting several cytoplasmic extensions to deliver oxygen. Their branched morphology is known to be plastic during development, allowing adaptation to tissue needs ${ }^{10}$. This tracheal branching phenomenon is reminiscent of angiogenic sprouting in vertebrates. Little is known about TTC plasticity in the adult fly and how it participates in tissue homeostasis and regeneration.

Perochon et al. and Tamamouna et al. now present evidence that local remodelling and extra branching of TTCs is important for the stem cell proliferative response upon intestinal damage (Fig. 1). Tracheal remodelling was found to accompany the well-described increase in ISC proliferation in response to damage by pathogenic bacteria, chemicals or oxidative agents and tumour growth. The authors could further show that increased branching of TTCs is highly dynamic and reversible, does not involve TTC cell division, and precedes changes in ISC proliferation and gut regeneration. Interestingly, the adaptability of the trachea to the microenvironment did not require ISC proliferation, as blocking proliferation through knockdown of myc in ISCs did not perturb tracheal branching ${ }^{5}$. In contrast, tracheal remodelling, or at least oxygen delivery, seemed to be essential for gut regeneration as hypoxic conditions or induced apoptosis of TTCs led to a loss of ISC proliferation after damage.

In Drosophila larvae, tracheal branching is controlled by hypoxia-driven induction of HIF-1 $\alpha /$ Sima, which induces expression of a Bnl/FGF ligand in the targeted tissue and then activates Btl/FGFR in the trachea, leading to its remodelling ${ }^{11}$. Using classical genetic approaches, the authors found that similar participants are involved in back-and-forth communication between the adult gut and trachea. Both studies found that HIF-1 $\alpha /$ Sima expression was induced upon damage in TTCs, whereas Tamamouna et al. noted an additional induction of HIF1 $\alpha /$ Sima in the gut epithelium. Similarly, $b t l / F G F R$ was upregulated in the trachea after damage, whereas $b n l / F G F$ was induced in the midgut cells and trachea. However, the genetic data indicate that $b t / / F G F R$ and bnl/FGF function in both gut epithelial cells and TTCs. Using loss- and gain-of-function approaches in a cell-type-specific manner (in TTCs, ISCs/enteroblasts (EBs), and enterocytes (ECs)), sima and $b t l$ were demonstrated to be necessary in TTCs for their remodelling and induction of ISC proliferation after damage. Despite the lack of an obvious increase of expression in gut epithelial cells, $b t l$ knockdown using esg-Gal4 (ISCs/EBs) or Myo1-Gal4 (ECs) also impaired branching and ISC proliferation. Nevertheless, the exact roles of $b n l$ and $b t l$ in the midgut cells, as well as potential differences in paracrine and autocrine signalling, remain to be elucidated. Indeed, even though Tamamouna et al. showed that $b t l$ and $b n l$ knockdown in ISCs and ECs affected both TTC branching and ISC proliferation, Perochon et al. observed that these treatments reduced ISC proliferation, but did not detect changes in tracheal branching. These differences may be due to the strength of the knockdown approach, perhaps suggesting that subtle differences in protein levels may have important physiological consequences. Altogether, both studies illustrate the 
High tracheal branching

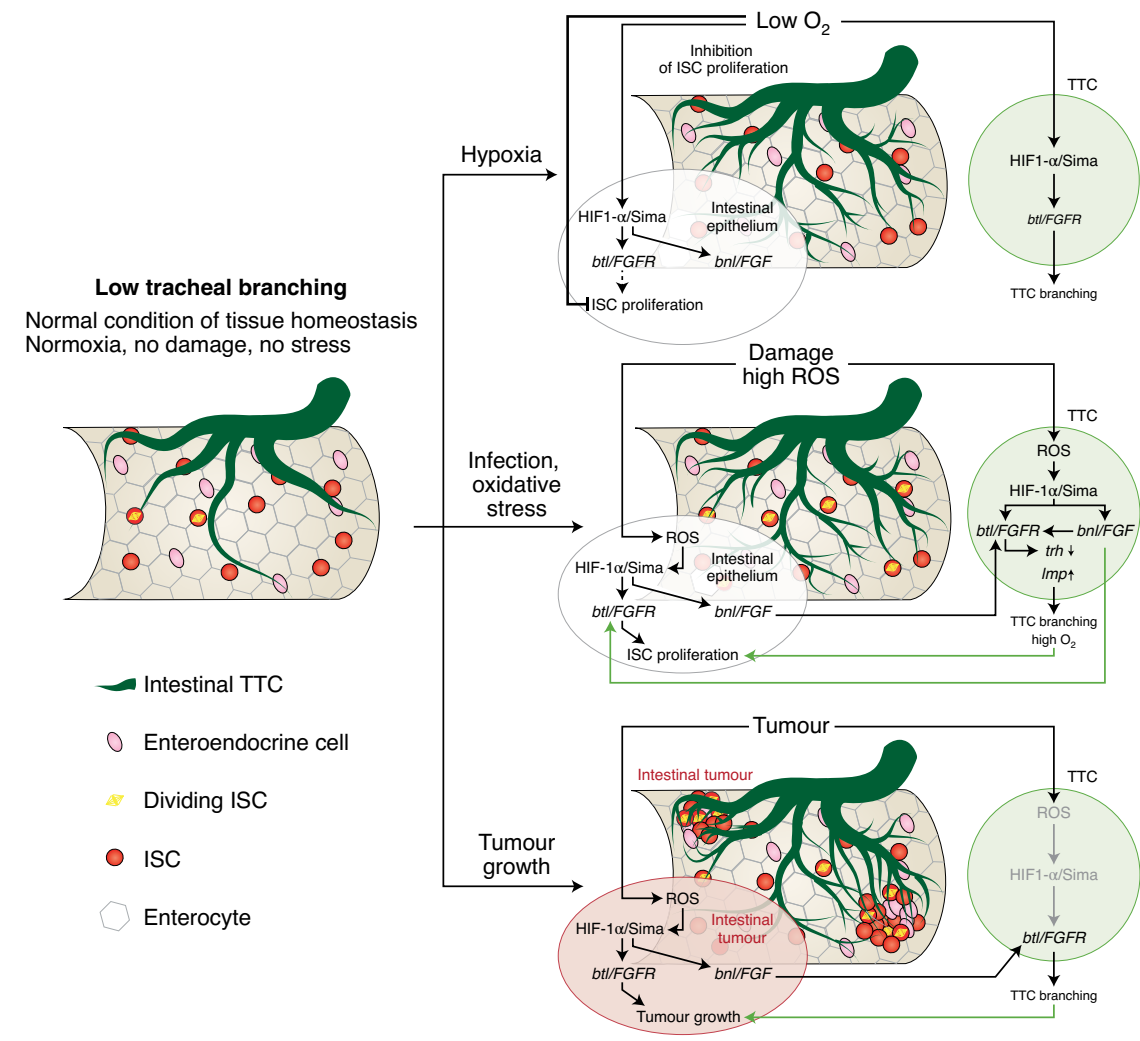

Fig. 1 | Schematic of the dialogue between the intestinal epithelium and the tracheal system in homeostasis, infection, cancer and hypoxia. In tissue homeostasis, intestinal stem cells (ISCs) are slowly dividing, balancing turnover of differentiated cells while the terminal tracheal cells (TTCs) provide oxygen $\left(\mathrm{O}_{2}\right)$ to the intestine. During hypoxia, the lack of oxygen induces HIF1 $\alpha /$ Sima activation and branchless/Fibroblast Growth Factor (bnl/FGF) expression in the midgut, allowing increased TTC coverage after Breathless/Fibroblast Growth Factor Receptor (Btl/FGFR) pathway activation. In the epithelium, the absence of sufficient oxygen distribution in hypoxic conditions blocks ISC proliferation and tissue regeneration after damage. After bacterial infection or oxidative stress, accumulation of reactive oxygen species (ROS) induces the communication between the trachea and intestinal epithelium, ultimately resulting in ISC proliferation and tracheal branching to provide extra oxygen to the tissue. Both processes involve ROS-induced HIF-1 $\alpha$ /Sima and Bt//FGFR signalling and are necessary for gut regeneration. Strikingly, intestinal tumour development involves the same factors active during the damage response. ROS production likely induces HIF1 $\alpha$ /Sima and Btl/FGFR pathway activation, allowing TTC extra-branching and tumour growth. Note, interactions in gray were not experimentally demonstrated but are hypothesized. Imp, IGF-II mRNA-binding protein.

importance of communication between the trachea and the gut epithelia to reshape the trachea and adapt ISC proliferation during regeneration (Fig. 1).

Interestingly, although hypoxia was shown to induce tracheal branching in the adult gut, it concomitantly blocked ISC mitosis, probably because oxygen is required for ISC proliferation (Fig. 1). As reactive oxygen species (ROS) have been implicated in the activation of hypoxia-inducible factor- $1 \alpha /$ Similar (HIF- $1 \alpha /$ Sima), the authors tested whether ROS production might be involved in tracheal remodelling and ISC proliferation upon stress. Feeding flies with the antioxidant $N$-acetyl cysteine (NAC) reduced $H I F-1 \alpha /$ sima and $b t l /$ FGF expression after damage, arguing that expression is ROS dependent ${ }^{5}$. Furthermore, infecting flies with bacteria mutants that could not produce pyocyanin, a ROS-generating secreted virulence factor, prevented TTC branching and ISC proliferation ${ }^{6}$, suggesting ROS involvement in both processes. Through genetic manipulation of ROS levels, the authors demonstrated that ROS production in both tissues is sufficient to induce tracheal branching and ISC proliferation. Again, this finding illustrates the cooperation between tracheal and gut tissues, which facilitates the adaption to tissue requirements, delivering increased oxygen and compensating for cell death during regeneration. It is worth noting how similar mechanisms of TTC branching are involved in developing and adult Drosophila after hypoxia ${ }^{11}$ and in response to ROS production. The conservation of angiogenic factors HIF- $1 \alpha$, FGF, FGFR and ROS in vascularization in vertebrates ${ }^{12}$ and described here in Drosophila is striking.

Perochon et al. ${ }^{5}$ identified genes with changed expression in the TTCs during remodelling using DNA adenine methyltransferase identification sequencing (DamID-seq) to evaluate genes differentially bound by RNA Pol II after bacterial infection. The conserved mRNA-binding protein Imp/IGF2BP, known to be involved in axonal remodelling in flies, was upregulated in TTCs in a ROS-dependent manner. Furthermore, Perochon et al. demonstrated that Imp/IGF2BP expression is required for both TTC remodelling and ISC proliferation upon damage. Surprisingly, TTC branching after damage also involved the downregulation of trachealess ( $t r h$ ), a transcription factor essential for the specification of tracheal fate during development. $t r h$ is believed to initiate or to act very early in the specification of tracheal cells during development ${ }^{10}$ Therefore, it is somewhat counterintuitive that $t r h$ should be downregulated in damaged TTCs to allow for proper branching and ISC proliferation. The authors propose that TTCs might dedifferentiate due to the downregulation of $t r h$, which may be important for allowing more plasticity and reshaping. Such a hypothesis is intriguing and merits further investigation into the mechanism behind trh downregulation, its role in TTC plasticity, and mammalian conservation during sprouting angiogenesis.

Neo-angiogenesis participates actively in tumour development and metastasis, providing oxygen and nutrients required for tumour growth and invasion. Counteracting angiogenesis has become a therapeutic strategy against cancer. Tamamouna et al. ${ }^{6}$ investigated the role of neo-tracheogenesis in intestinal tumour growth. Inducing ISC-derived tumours expressing an activated form of Ras $\left(\mathrm{Ras}^{\mathrm{V} 12}\right)$ or inactivating Notch in ISCs, they observed increased tracheation locally. In accordance with previous studies ${ }^{13}$, transplantation of tumour cells induced the recruitment of tracheal cells around the transplant. The excess tracheal branches seemed to be important for tumour growth and survival, as both were strongly reduced in hosts in which $b t l$ was knocked down. Mechanistically, $\mathrm{Ras}^{\mathrm{V} 12}$ tumours induced $b t l, b n l$ and sima 
expression and knockdown of these factors in the tumour reduced tracheal branches and tumour size and increased fly survival. Prevention of ROS production in the tumour reduced tracheal coverage, ISC mitosis, and tumour growth. However, it remains difficult to dissociate a ROS function on vascularization of the tumour from previously described roles of ROS directly on stem cell proliferation ${ }^{14,15}$. Nevertheless, the conservation of mechanisms and factors involved in damage response and upon tumorigenesis in the fly intestine and neo-angiogenesis is evident.

An interesting finding reported by both groups is the reversibility of tracheogenesis after epithelial damage ${ }^{5}$ or upon tumorigenesis ${ }^{6}$ (after switching off $\mathrm{Ras}^{\mathrm{V} 12}$ expression). Thus, a future aim for the field will be to better understand mechanisms and factors regulating trachea plasticity during this recovery phase concomitant with the slowdown of stem cell proliferation.

In summary, both studies establish the Drosophila intestine as an excellent example of tissue collaboration regulating adult stem cells, tissue regeneration or tumour growth. Future studies using this model will provide a better understanding of the mechanisms required for co-regulation and dynamic plasticity of vascular tissues and stem cell responses.

Louis Gervais ${ }^{\bowtie}$ and Allison J. Bardin (D) $₫$ Institut Curie, PSL Research University, CNRS UMR 3215, INSERM U934, Stem Cells and Tissue Homeostasis Group, Paris, France.

$凶_{e}$-mail:louis.gervais@curie.fr; allison.bardin@curie.fr

Published online: 26 May 2021

https://doi.org/10.1038/s41556-021-00695-w
References

1. Ding, L., Saunders, T. L., Enikolopov, G. \& Morrison, S. J. Nature 481, 457-462 (2012).

2. Kiel, M. J. et al. Cell 121, 1109-1121 (2005).

3. Shen, Q. et al. Cell Stem Cell 3, 289-300 (2008).

4. De Palma, M., Biziato, D. \& Petrova, T. V. Nat. Rev. Cancer 17, 457-474 (2017).

5. Perochon, J. et al. Nat. Cell Biol. https://doi.org/10.1038/s41556021-00676-z (2021).

6. Tamamouna, V. et al. Nat. Cell Biol. https://doi.org/10.1038/ s41556-021-00674-1 (2021)

7. Gervais, L. \& Bardin, A. J. Curr. Opin. Cell Biol. 48, 97-105 (2017).

8. Li, Z., Zhang, Y., Han, L., Shi, L. \& Lin, X. Dev. Cell 24 133-143 (2013).

9. Linneweber, G. A. et al. Cell 156, 69-83 (2014). 10. Hayashi, S. \& Kondo, T. Genetics 209, 367-380 (2018).

11. Centanin, L. et al. Dev. Cell 14, 547-558 (2008).

12. Fraisl, P., Mazzone, M., Schmidt, T. \& Carmeliet, P. Dev. Cell 16, 167-179 (2009).

13. Campbell, K. et al. Nat. Commun. 10, 2311 (2019).

14. Hochmuth, C. E., Biteau, B., Bohmann, D. \& Jasper, H. Cell Stem Cell 8, 188-199 (2011).

15. Patel, P. H. et al. Nat. Commun. 10, 4365 (2019).

Competing interests

The authors declare no competing interests. 